\title{
A Simple and Effective Geometric Representation for Irregular Porous Structure Modeling
}

\author{
X.Y. Kou and S.T. Tan* \\ Department of Mechanical Engineering, The University of Hong Kong, Pokfulam Road, Hong Kong
}

Please cite the article as follows:

Kou, X.Y. and S.T. Tan, A simple and effective geometric representation for irregular porous structure modeling. Computer-Aided Design, 2010. 42(10): p. 930-941.

Youtube Video Link:

http://www.youtube.com/watch?v=12PahzCUrAI

Related Article:

X.Y. Kou, S.T. Tan, Modeling Functionally Graded Porous Structures with Stochastic Voronoi Diagram and B-Spline Representations, 2010 International Conference on Manufacturing Automation, Hong Kong, December 13-December 15, 2010, http://doi.ieeecomputersociety.org/10.1109/ICMA.2010.36

* Corresponding author. Tel.: +852-2859-7909; fax: +852-2858-5415.

E-mail addresses: kouxy@hku.hk (X.Y. Kou), sttan@hku.hk (S.T. Tan). 


\title{
A Simple and Effective Geometric Representation for Irregular Porous Structure Modeling
}

\author{
X.Y. Kou and S.T. Tan* \\ Department of Mechanical Engineering, The University of Hong Kong, Pokfulam Road, Hong Kong
}

\begin{abstract}
Computer-aided design of porous structures is a challenging task because of high degree of irregularity and intricacy associated with the geometries. Most of the existing design approaches either target at designing artifacts with regular-shaped pores or reconstructing geometric models from existing porous objects. For regular porous structures, it is difficult to control the pore shapes and distributions locally; for reconstructed models, a design is attainable only if there are some existing objects to reconstruct from. This paper is motivated to present an alternative approach to design irregular porous artifacts with controllable pore shapes and distributions, yet without requiring any existing objects as prerequisites. Inspired by the random colloidaggregation model which explains the formation mechanism of random porous media, Voronoi tessellation is first generated to partition the space into a collection of compartments. Selective compartments are then merged together to imitate the random colloid aggregations. Through this Voronoi cell merging, irregular convex and concave polygons are obtained and the vertices of which are modeled as control points of closed BSpline curves. The fitted B-Spline curves are then employed to represent the boundaries of the irregular-shaped pores. The proposed approach drastically improved the ease of irregular porous structure modeling while at the same time properly maintained the irregularity that are widely found in natural objects. Compared with other existing CAD approaches, the proposed approach can easily construct irregular porous structures which appear more natural and realistic.
\end{abstract}

Keywords: Nature inspired design, Porous structure, Porosity, CAD, Voronoi Diagram, Spline, Random aggregate.

\section{Introduction}

Computer-aided design of porous objects is getting increasingly important in recent years. This popularity is largely driven by the wide application of porous artifacts where porous objects manifest many appealing or superior properties to solid counterparts. For instance, porous objects usually have larger internal surface areas and higher strength-to-

* Corresponding author. Tel.: +852-2859-7909; fax: +852-2858-5415.

E-mail addresses: kouxy@hku.hk (X.Y. Kou), sttan@hku.hk (S.T. Tan). 
weight ratios [1, 2], interconnected pores can facilitate the passage of fluids and biological cells, upon which biological tissues can be gradually grown and docked.

In the past few decades, considerable research efforts in porous object design have been reported [1, 3-13]. There have been targeted approaches to design regular porous objects [5-8, 14] (e.g. truss-like structures) and methods to reconstruct geometric models that resemble natural porous objects (e.g. bones [9, 10] and sandstones [11-13]).

Regular objects are generally easier to model and fabricate, however, it is often difficult, if possible, to exert local control on the pore sizes and pore distributions, therefore the design freedom and general applicability of such approaches are often overrestricted. The reconstructed geometric models look more natural and realistic and many of such models include irregular-shaped pores with non-uniform pore distributions. It is generally believed that such complex structures can provide optimized mechanical, thermal or other physical responses under specific loading conditions [15]. Designing similarly structured objects by means of replication/mimicry or $3 \mathrm{D}$ reconstruction is therefore an effective and appealing approach to derive functionally equivalent objects with optimized performances. However, it should be noted that such optimality can be assured only when the reconstructed model $A$ and the object to mimic $B$ are subject to the same stimuli and constraints. In scenarios where the external environment of $A$ is different from the natural counterpart $B$, exact reconstruction from existing objects is not quite useful and effective. Moreover, reconstruction based approaches assume some existing porous objects are always available at hand which significantly limited their efficacy and usefulness.

The primary goal of this paper is to propose an alternative nature-inspired geometric representation for irregular porous structure modeling, with which porous artifacts can be effectively constructed, as opposed to being reconstructed conventionally. The emphasis of this paper is therefore twofold: to present geometric representation for modeling irregular porous structures and to design such artifacts from a constructive perspective.

In Section 2, we briefly review pertinent work in literature and discuss the motivations of this work in more details. Section 3 presents a hybrid Voronoi tessellation and BSpline based representation for modeling irregular porous structures. Based on the proposed representation, several case studies are offered in Section 4 to show the efficacies and flexibilities of the proposed approach. Section 5 discusses the pros and cons of this study and concluding remarks are offered at the end.

\section{Related work and motivations}

\subsection{Related work}

The study of regular porous structures can be dated back to 17's century when Robert Hooke (who coined the word "cell") first examined cork's microstructure with his 
microscope [16]. Since then, mathematicians and engineers were particularly fascinated by the regularity that appeared in the honeycomb structures.

In the past few decades, some efforts attempted to use mature solid modeling techniques to design internal geometries of the objects. An overwhelmingly used approach is to design the shape of a unit cell and then apply certain types of (e.g. linear or circular) patterns to form periodic structures in space, as exemplified in Fig. 1. Hollister et al $[2,17]$ modeled the unit cell structure composed of intersecting cylindrical pores and assumed the porous structure under design is locally periodic. Hutmacher [18] used rectangles and triangles to represent the pore geometries, and linear patterns are applied to form regular non-woven constructs. Lal and Sun [19] proposed microsphere packed three-dimensional bone grafts in their scaffold design. Chua et al [5] investigated the use of polyhedral shapes, inclusive of Platonic, Archimedean solids, prisms and anti-prisms etc. as the unit cell matrices.

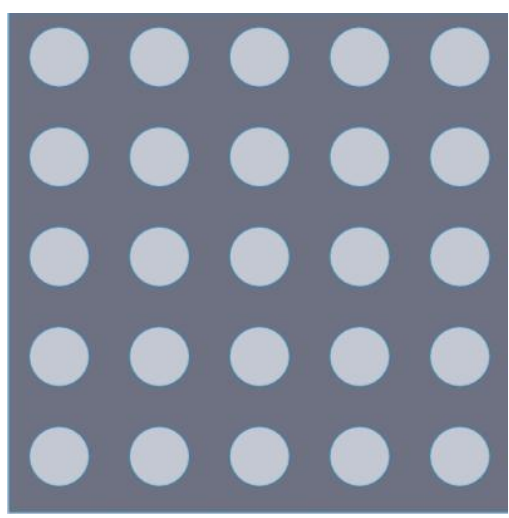

(a)

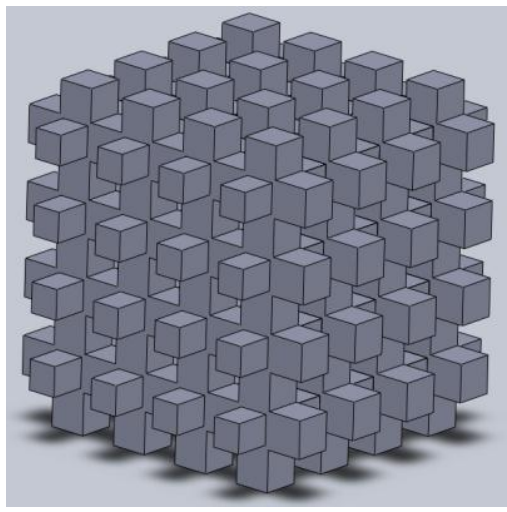

(b)

Fig. 1 Examples of regular porous object models.

The advantages of periodic porous structures lie in their ease of modeling, fabrication [18, 20, 21] as well as the property evaluation and prediction [20, 22, 23]. Using standard mechanics Representative Volume Element (RVE) based analyses or homogenization methods [14, 15, 22-29], the effective properties of the unit cells can be computed from the structural analysis at microscopic levels. Hollister and Kikuchi [15] compared the homogenization and standard mechanics RVE based analysis for periodic porous composites, and they showed homogenizataon theory is preferable over standard mechanics for periodic composites. Fang et al [22], McIntosh et al [20] and Khatam and Pindera [30] applied the homogenization methods to characterize the effective structural properties (e.g. Poisson's ratio and Young's modulus). Porfiri et al [31] derived estimates for effective thermal conductivity of hollow particle filled composites.

Despite the straightforwardness in modeling and analysis, the pore shapes of the above discussed structures are mostly regular (such as triangular, square, rectangular, pentagonal or hexagonal) primitives [2]. To model more intricate unit cells, Sun et al [7] and Fang et al [22] introduced a library of complex primitives including the woven, 
braided and knit features and the unit cells are no longer constrained to the simple geometries such as spheres or triangles. Instead of relying on designers and CAD tools to design unit cell geometries, Lin et al [32] proposed to use topology optimization based approach to design the void and solid material for a unit cell. Given an initial randomly distributed solids and voids, the unit cell's effective properties are evaluated using homogenization methods. Sensitivity derivatives of objective functions with respect to the design variables are then calculated, and subsequent iterations are performed to update the configurations of solids and voids. Such processes are repeatedly carried out until the unit cell's geometries converge. Note that even though the unit cell's geometry can be relatively more irregular in these cellular models, however, these cells are still regularly and repeatedly packed in the geometric domain. Due to such regularity and periodicity, it is very difficult to exert local control on the pore shapes, sizes and distributions, since a minute modification on the unit cell will result in global changes in the entire internal structures.

Rather than packing the unit cells into linear, circular or other regular patterns, stochastic packing approaches can avoid such limitations. In such methods, the anchors of the unit cell are generated from stochastic processes. Schroeder et al [33] discussed the use of random point process, for instance, the Poisson process to specify the centers of spheres, and the collection of spheres are modeled as pores by applying Boolean subtraction from the external geometries. Luchnikov et al [34] proposed to embed nonspherical convex shapes (e.g. spherocylinders, i.e. cylinders with hemisphere at the ends) into Voronoi $S$ regions (extension of Voronoi polyhedral with curved faces and edges). Using such arrangement, the designed porous structures can have more irregular pore distributions. The modification on any single pore will not propagate into the entire porous structure, and local control is therefore attainable to a flexible degree.

Besides the aforementioned constructive approaches, reverse-engineering based methods are also extensively used in porous structure modeling. Porous object models reconstructed from a number of sources were well studied, these include magnetic resonance imaging (MRI), scanning electron microscope (SEM), energy dispersive spectroscopy, Fourier transform IR spectroscopy, and X-ray computed tomography (CT) [35-39] etc, as demonstrated in Fig. 2.

Virnovsky et al [40] modeled the void space of porous rock at capillary equilibrium, a bundle of capillary tubes of arbitrary cross-sections taken directly from photomicrographs were used to represent irregular pores. They demonstrated that such reconstructed models can retain important features of realistic pore space geometry, curvature and roughness. Tsakiroglou et al [37] developed an "inverse modeling" method for pore structure characterization by combining experimental intrusion/retraction curves with Back-scattered Scanning Electron Microscope (BSEM) images, a realistic representation of the pore space of dual porosity rock was derived. Mohebi et al [36] proposed a statistical fusion framework for reconstructing high-resolution porous media 
from low-resolution measurements, a posterior sampling based approach was presented to combine low-resolution MRI/X-ray measurements with high-resolution microscopic data. Levy et al [41] acquired spiral CT data with different resolutions and the data were converted to vector file format. By cross-hatching of the vector file data, 2D porous structures were obtained and subsequently used for prosthesis manufacture on rapid prototyping machines.

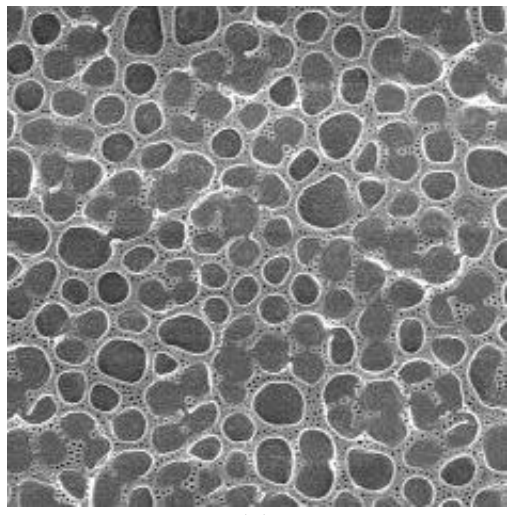

(a)

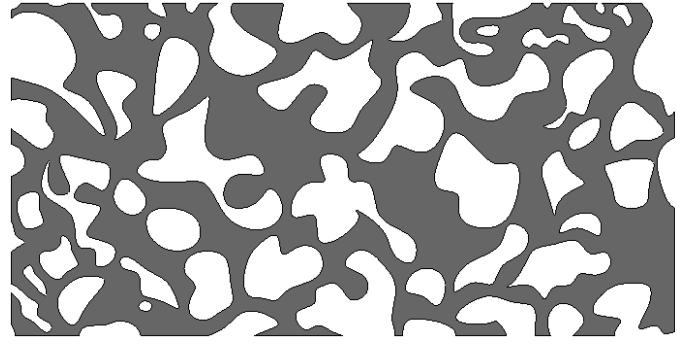

(b)

Fig. 2 Examples of irregular porous structures. (a) and (b) are adapted from [38] and [39] respectively.

\subsection{Motivations of this research}

From the brief review above, it can be seen that the majority of constructive CAD modeling approaches are targeted at designing regular porous structures while most of irregular porous objects are designed by reconstructive approaches. The regular porous objects here refer to those which are modeled with simple and primitive unit cells or those designed by packing the unit cells into linear, circular or other regular patterns. Such "highly simplified geometrical structures" [42] are generally easier to model, visualize, analyze and fabricate, however, it is difficult to exert local control on the pore sizes and pore distributions. Moreover, such models are unnatural, unrealistic and many porous structures commonly seen in nature cannot be easily represented.

In terms of representational fidelity, the reconstructed porous object models perform much better than the regular ones. They are generally characterized as having irregularly shaped pores, non-uniform and random pore distributions, and there are no specific fixed priori imposed on the internal geometries. However, the reconstruction based approaches assume some existing porous objects are always available at hand otherwise it will be impossible to generate high quality models that can describe the internal geometries, such as those shown in Fig. 2. In addition, exact imitation or reconstruction of existing porous structure is not always effective and penalty-free. The complex microstructures of existing porous structure in nature such as wood and human bone tissues are known to be

optimized for withstanding functional loads they are subject to [15], however when using such reconstructed models, the external environments have rarely been quantified, 
included and closely associated with the optimized models. In scenarios where the external environment (for instance, the magnitude and distributions of the external stimuli) of the object is different from that of the object under design, such reconstructed models will be inadequate to guarantee optimized performances.

What this paper is trying to address is to propose a geometric representation to model such irregular porous structure as shown in Fig. 2, and to design such artifacts from a constructive perspective from scratch. Instead of designing a faithful copy from 3D reconstruction, the proposed method does not require any pre-existing models, yet it can generate generically similar structures with irregular pore shapes and distributions.

\section{A nature inspired geometric representation for irregular porous structure modeling}

As noted in previous section, the objective of this study is to design irregular porous structures such as those shown in Fig. 2. Although it is straightforward to explicitly model the pore boundaries with BSpline or NURBS curves, however, manually design such cavities via direct specification of control polygons/polyhedrons are extremely tedious and unwieldy. Moreover, the modeled pore shapes and pore distributions would be highly dependent on or considerably constrained by designer's creativity and experience. In this paper, we propose a nature-inspired representation which imitates the formation process of porous structure.

\subsection{Random aggregation model}

In their seminal work, Schaefer and Keefer [42] for the first time, proposed a model that explained the formation mechanism of random porous materials. They contended that the porosity is due to random "jungle gym" or branched-polymer-like structure [42]. From experimental data derived from small-angle x-ray scattering (SAXS), they proposed a random colloid-aggregation model which states that during polymerization, "the silica particles first grow to a mean radius and then aggregate in a ramified manner and ultimately gel" [42].

Inspired by Schaefer and Keefer's random colloid-aggregation model, in this paper, we propose a geometric representation that mimics the physical/chemical process of polymerization. Voronoi tessellation is first generated to partition the space into a collection of compartments. Selective compartments are then merged together to imitate the random colloid aggregations. Through this Voronoi cell merging, irregular convex and concave polygons are obtained and the vertices of which are modeled as control points of closed BSpline curves. The fitted B-Spline curves are then employed to represent the boundaries of the irregular-shaped pores. 


\begin{tabular}{l|l}
\hline Physical/Chemical process & Nature inspired counterpart \\
\hline Polymerization & Porous structure formation/modeling \\
Jungle gym & Voronoi tessellation \\
Ramifying process & Attribute based branching/classification \\
Colloid aggregation & Voronoi Cell/polygon merging \\
\hline
\end{tabular}

Tab. 1 An analogy of physical/chemical polymerization and the nature inspired counterpart

The analogy between the terms/processes in Schaefer-Keefer model and the corresponding CAD counterparts are briefly listed in Tab. 1, and each mimetic modeling process is detailed in what follows.

\subsection{A hybrid Voronoi tessellation and B-Spline based representation for pore shape and pore distribution modeling}

To implement the "Jungle gym" structure, Voronoi tessellation is first generated to partition the space into a collection of compartments. For a point set $P=\left\{p_{1}, p_{2}, \cdots, p_{n}\right\}, n \geq 2$ in the $m$-dimensional space $R^{m}$, the region $V\left(p_{i}\right)$ associated with a Voronoi generator point $p_{i}$ which follows Eq. (1) is defined as the Voronoi cell of $p_{i}[43]$ :

$$
V\left(p_{i}\right)=\left\{x \mid\left\|x-x_{i}\right\| \leq\left\|x-x_{j}\right\| \text {, for } i \in I_{n}, j \in I_{n}, j \neq i\right\}
$$

where $\left\|x_{1}-x_{2}\right\|$ is the Euclidean-distance between point $x_{1}$ and $x_{2}, I_{n}=\{1,2, \cdots, n\}$. Intuitively, the Voronoi cell or Voronoi region $V\left(p_{i}\right)$ consists of a point set, the elements of which are closer to $p_{i}$ than to any other points in $P$. The $m$-dimensional space $R^{m}$ is partitioned into exactly $n$ regions $v=\left\{V\left(p_{1}\right), V\left(p_{2}\right), \cdots, V\left(p_{n}\right)\right\}$, as demonstrated in Fig. 3 (a), and this partition is called the Voronoi diagram of $P=\left\{p_{1}, p_{2}, \cdots, p_{n}\right\}$ and $P$ is called the generator points of this Voronoi diagram.

In $R^{2}$, the boundaries of Voronoi cells contain line segments which are the perpendicular bisector of the line that joins two associated generator points. The boundary is called Voronoi edge, and all the Voronoi edges of the same Voronoi cell form a Voronoi Polygon. The point where three or more Voronoi edges meet is called Voronoi point or Voronoi Vertex.

Given a set of distinct points $P=\left\{p_{1}, p_{2}, \cdots, p_{n}\right\}, n \geq 2$, the Voronoi diagrams can be constructed using Fortune's plane-sweep algorithm [44]. Let $V\left(p_{i}\right)$ be a Voronoi cell associated with the generator $p_{i}$ and the vertices of the Voronoi polygon $V\left(p_{i}\right)$ be $\left\{v_{0}, v_{1}, \cdots v_{m}\right\}$, if we regard these Voronoi vertices as the control points of a closed BSpline curve $C(u)$ of degree $k$, then 


$$
C(u)=\sum_{i=0}^{m} N_{i, k}(u) v_{i}
$$

where $N_{i, k}(u)$ is B-Spline basis function of degree $k$, which can be recursively defined by Eq. (3). To create a periodic B-Spline curve, the first and last $k$ control points must be properly wrapped in the form such that $v_{0}=v_{m-k+1}, v_{1}=v_{m-k+2}, \ldots, v_{k-2}=v_{m-1}$ and $v_{k-1}=v_{m}$.

$$
\begin{aligned}
& N_{i, 0}(u)= \begin{cases}1 & \left(u_{i} \leq u<u_{i+1}\right) \\
0 & (\text { otherwise })\end{cases} \\
& N_{i, k}(u)=\frac{u-u_{i}}{u_{i+k}-u_{i}} N_{i, k-1}(u)+\frac{u_{i+k+1}-u}{u_{i+k+1}-u_{i+1}} N_{i+1, k-1}(u)
\end{aligned}
$$

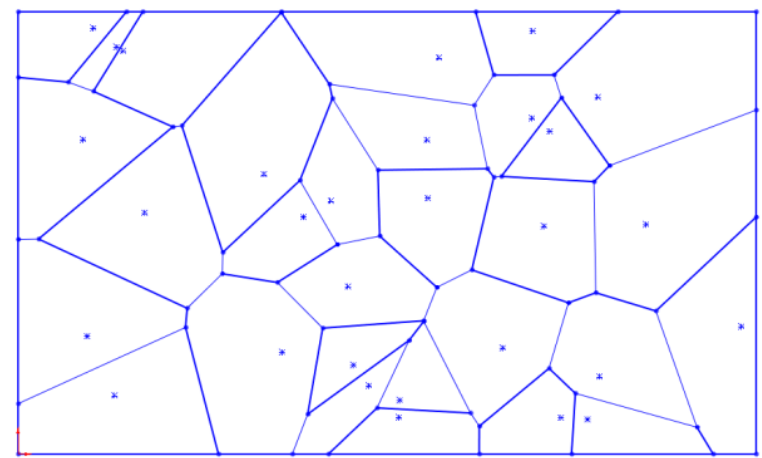

(a)

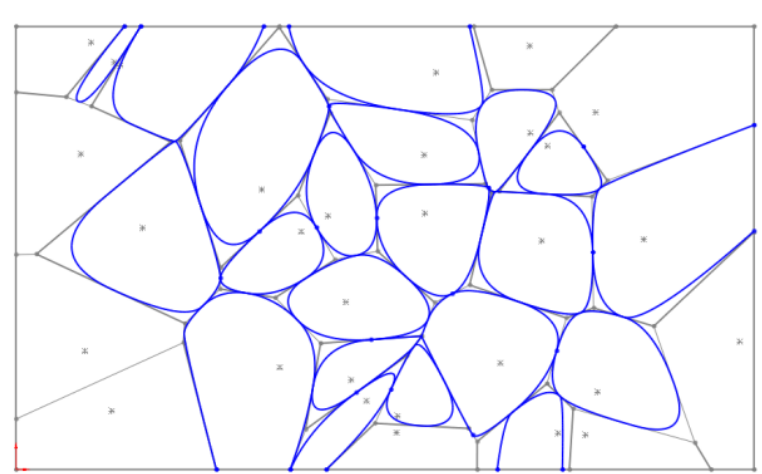

(b)

Fig. 3 Voronoi Diagram and B-Spline curves fitted from Voronoi polygon vertices

Fig. 3 (b) shows some cubic periodic B-Spline curves generated from the Voronoi cells shown in Fig. 3 (a). In this paper, we propose to use such closed B-Spline curve to represent the pore boundaries.

As can be seen from this figure, this hybrid Voronoi-BSpline based representation is very suitable to represent irregular pore shapes. Since there are unlimited numbers of Voronoi tessellations for $R^{m}$ and its subspaces, it is guaranteed that the shapes of the associated Voronoi polygons are not restricted to regular patterns. The use of periodic BSpline curves to represent the pore boundaries can also effectively characterize the real world pore geometries which are seldom polygon or polyhedra-like, as shown in Fig. 2.

This simple representation, however, also has some limitations. For instance, the interstices between adjacent pores are too small and many pores nearly touch each other. Moreover, it is known that the Voronoi cells generated from distinct points are always non-empty convex set [43], therefore it is impossible to model such porous structures as shown in Fig. 2 (b), for which concave features such as crease edges or corners are commonplace.

To control the interstices in between the modeled pores, a straightforward approach is to increase the order $k$ of the B-Spline curves, since the higher the order $k$ is, the farther 
the fitted curve deviates from the control polygon, as illustrated in Fig. 4. Although a high order can be used in B-Spline curve fitting, however it is less practical to infinitely increase the order $k$.

An alternative effective method is to scale the control polygon instead, and use the scaled polygon vertices to fit the B-Spline curves. In doing so, the centroid of the polygon is first computed from Eq.(4), which is used as the scaling center. The scaled polygon vertex can be then derived from Eq. (5):

$$
\begin{gathered}
x_{c}=\frac{1}{m} \sum_{i=1}^{m} x_{i}, y_{c}=\frac{1}{m} \sum_{i=1}^{m} y_{i} \\
x_{i}^{\prime}=x_{c}+t\left(x_{i}-x_{c}\right), y_{i}^{\prime}=y_{c}+t\left(y_{i}-y_{c}\right)
\end{gathered}
$$

where $\left(x_{c}, y_{c}\right)$ is the polygon centroid, $\left(x_{i}, y_{i}\right)$ is the $i$-th control vertex of the polygon, $\left(x_{i}^{\prime}, y_{i}^{\prime}\right)$ is the scaled control vertex, $0<t \leq 1$ is the scaling factor and $m$ is the total number of vertices of the control polygon. Fig. 5 shows the increments of pore interstices using scaled control polygons.

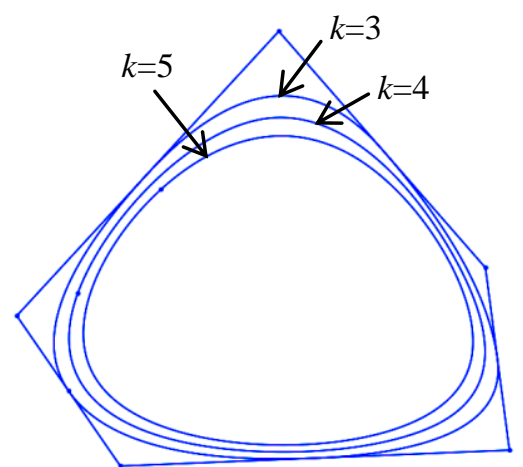

Fig. 4 Different orders of B-Spline curves and the control polygon.

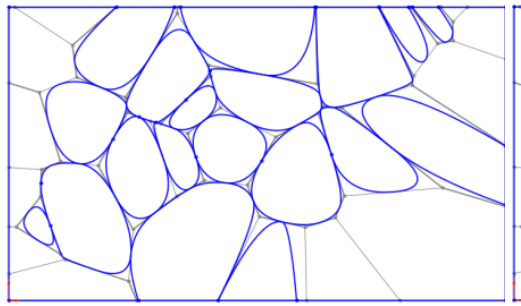

(a)

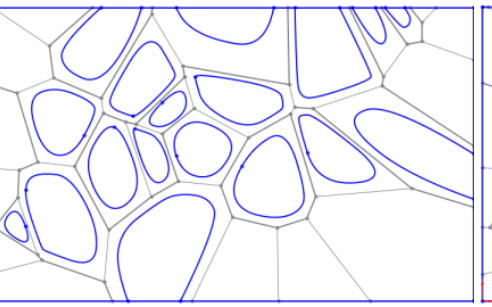

(b)

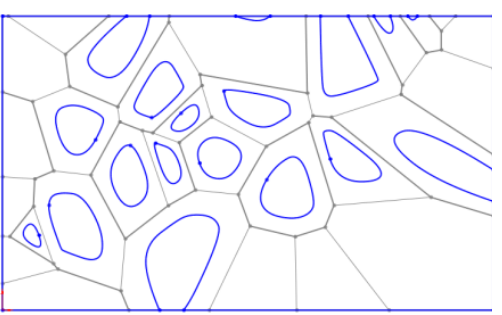

(c)

Fig. 5 Modelling increased pore interstices using scaled control polygons. (a) Scale factor $t=1.0$; (b) Scale factor $t=0.8$; (c) Scale factor $t=0.6$.

\subsection{Attribute association and Voronoi polygon aggregation}

To enable modeling pores with concave shapes, the most straightforward way is to introduce concave polygons as the control points of B-Spline curves. Inspired by the 
random colloid-aggregation model discussed in Section 3.1, we proposed to selectively merge some Voronoi polygons together so as to allow concave polygons to be modeled.

To accomplish this, an integer attribute $\lambda$ is associated to each Voronoi polygon as shown in Fig. 6 (a) and (b), here different colors are used to represent the attributes associated with different Voronoi cells. The attribute $\lambda$ is a random integer ranging from $\left[\lambda_{\min }, \lambda_{\max }\right]$ indicating the category it belongs to.

Two cells $A$ and $B$ are merged/aggregated together on the following conditions. (i) $A$ and $B$ belong to the same category, i.e. they have the same attribute value; and (ii) $A$ and $B$ are adjacent, i.e. they share some Voronoi edges in common.

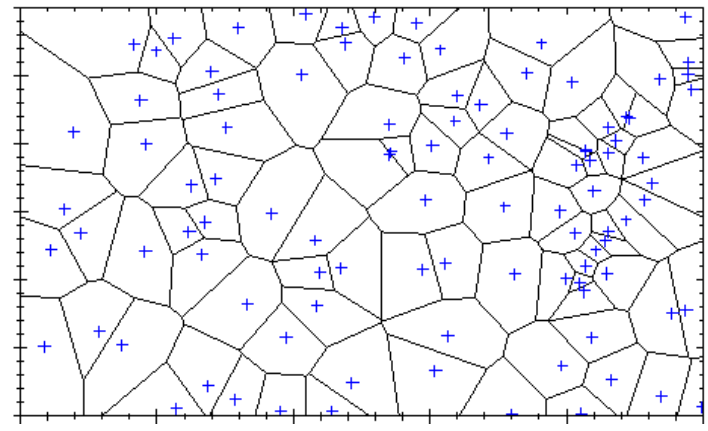

(a)

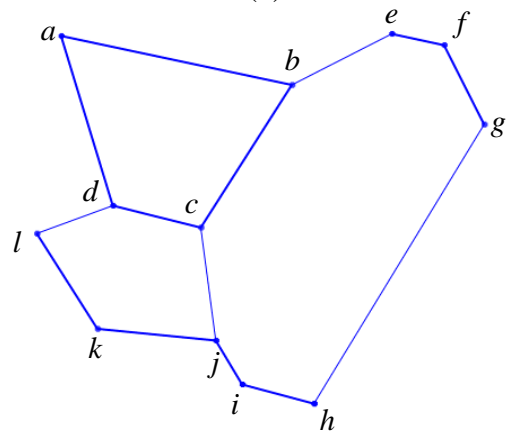

(c)

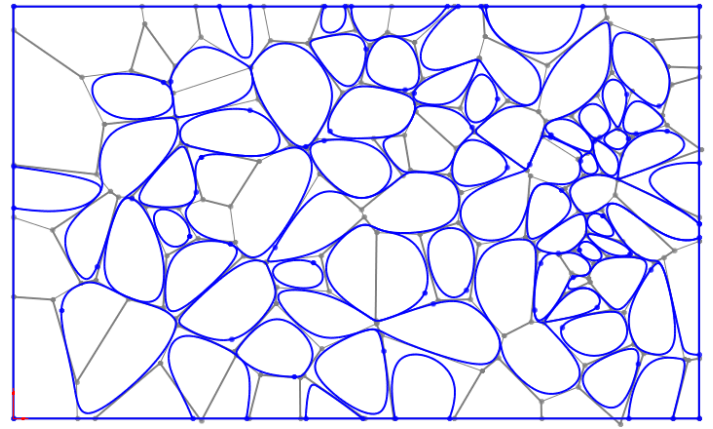

(e)

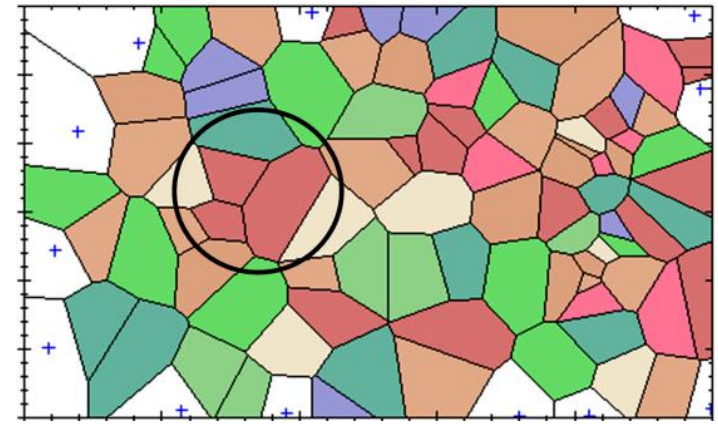

(b)

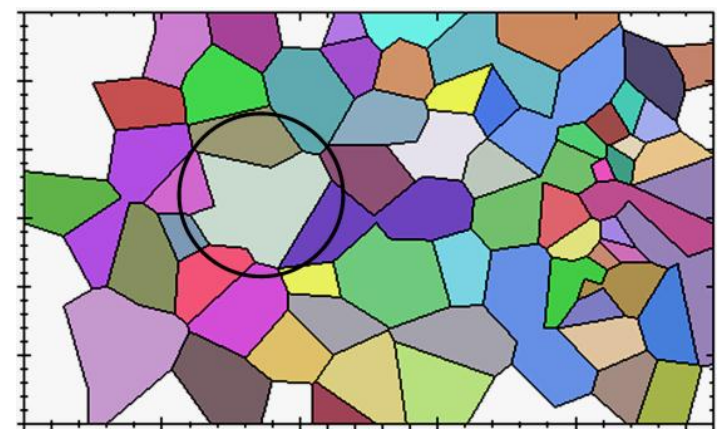

(d)

Fig. 6 Cell attributes association and concave pore modelling. (a) A Voronoi diagram with no attributes associated. (b) Each cell is randomly associated with an integer attribute, represented with a fill color. (c) A zoomed view of three Voronoi polygons to be merged. (d) Adjacent same-attributed Voronoi polygons are merged to form a bigger polygon. (e) The fitted B-Spline curves and their control polygons. Note that the cell attribute of the merged cell shown in (d) may not be exactly the same as the attributes of the child cells in (b), and attributes are used only for classification purposes only. 
Fig. 6 (b) highlights three Voronoi cells which satisfy these conditions (for brevity, we call them the child polygons of the merged polygon), and we will use the three cells inside the bounded circle as example to explain the polygon merging algorithm, as is shown in Fig. 7.

Let the Voronoi vertices of the three Voronoi cells in Fig. 6 (c) be $\{a, b, c, d\}$, $\{b, c, e, f, g, h, i, j\}$ and $\{c, d, j, k, l\}$, and the Voronoi edges of these polygons are:

$$
\begin{aligned}
v e_{1} & =\{a \leftrightarrow b, b \leftrightarrow c, c \leftrightarrow d, d \leftrightarrow a\} \\
v e_{2} & =\{b \leftrightarrow e, e \leftrightarrow f, f \leftrightarrow g, g \leftrightarrow h, h \leftrightarrow i, i \leftrightarrow j, j \leftrightarrow c, c \leftrightarrow b\} \\
v e_{3} & =\{c \leftrightarrow j, j \leftrightarrow k, k \leftrightarrow l, l \leftrightarrow d, d \leftrightarrow c\}
\end{aligned}
$$

where the symbol $x \leftrightarrow y$ denotes vertex $x$ is connected with vertex $y$, and $x$ and $y$ are the bounding vertices of an edge. To get the edges of the merged polygon, all the shared edges of the child polygons are excluded (L5, Fig. 7) while the remainder non-shared ones, which constitute the edges of the merged polygon, are appended to the merged edge list (L7, Fig. 7), and the sharing status is determined from the code shown in L4, Fig. 7.

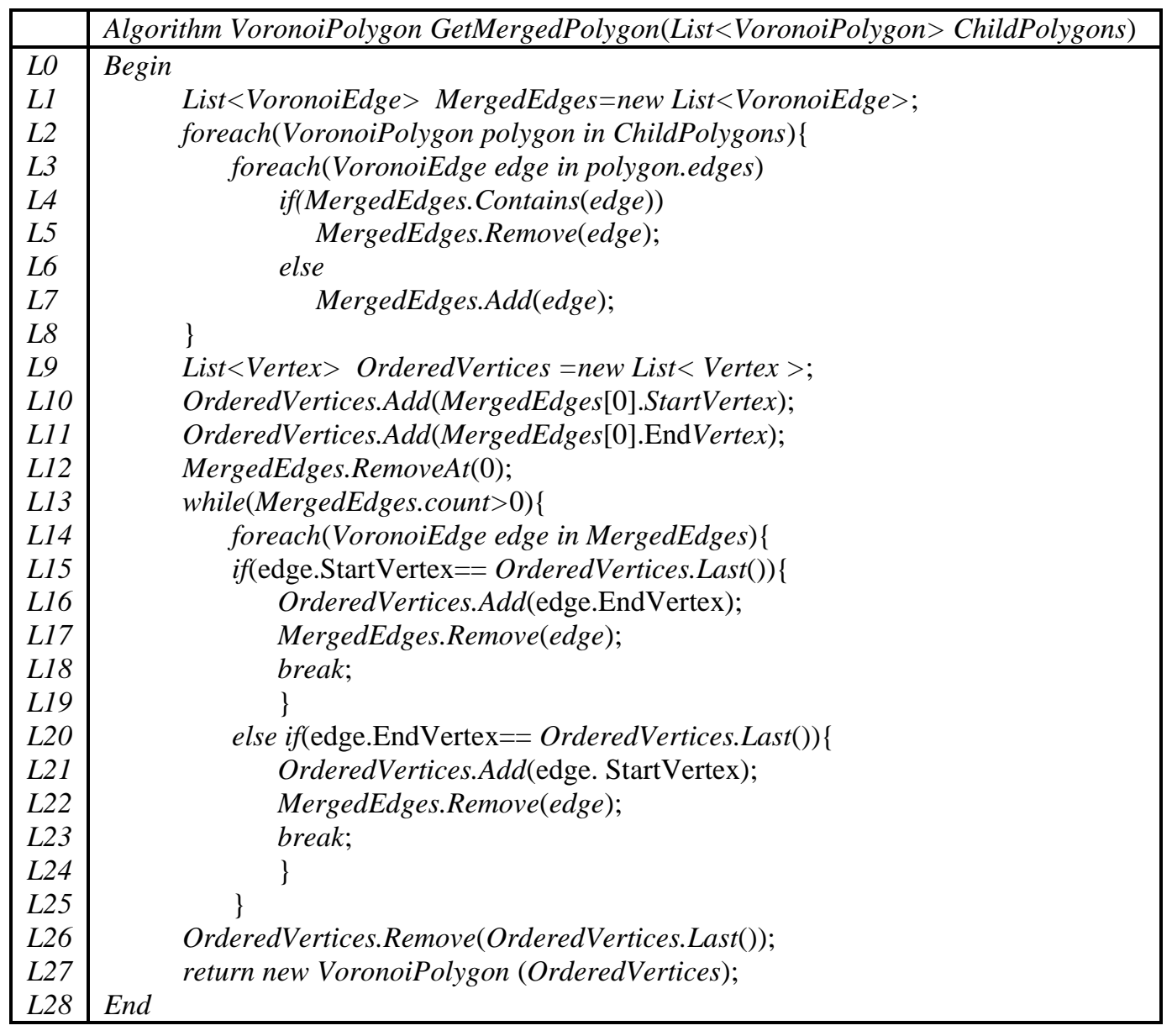

Fig. 7 Pseudo code of polygon merging algorithm. 
By definition $x \leftrightarrow y$ is equivalent to $y \leftrightarrow x$, the edge pairs $\left(b \leftrightarrow c \in v e_{1}, c \leftrightarrow b \in v e_{2}\right),\left(j \leftrightarrow c \in v e_{2}, c \leftrightarrow j \in v e_{3}\right)$ and $\left(c \leftrightarrow d \in v e_{1}, d \leftrightarrow c \in v e_{3}\right)$ in Eq.(6) are shared Voronoi edges and therefore excluded, and the edges of the merged polygon are therefore:

$$
e=\{a \leftrightarrow b, d \leftrightarrow a, b \leftrightarrow e, e \leftrightarrow f, f \leftrightarrow g, g \leftrightarrow h, h \leftrightarrow i, i \leftrightarrow j, j \leftrightarrow k, k \leftrightarrow l, l \leftrightarrow d\}(7)
$$

Note that the edges of the merged polygon are generally stored in random orders in memory and there is no guarantee that the adjacent edges in the list are incident to each other. For visualization purposes, this does not matter; however to fit closed B-Spline curves, the sequence of the edge vertices does make a difference. To rearrange the edges such that adjacent edges in the list are also geometrically consecutive, an ordered vertex list is created (L9, Fig. 7). All the vertices are then saved into the ordered list according to the edge connectivity relations.

The two vertices of first edge, i.e. $a$ and $b$ are first appended in the ordered list, and the next edge which is incident to the last vertex $b$, is then identified (L15, Fig. 7). In this example, the next edge is $b \leftrightarrow e$, so the vertex $e$ is appended (L16, Fig. 7) to the ordered list, and the edge $b \leftrightarrow e$ is excluded (L17, Fig. 7) since both its bounding vertices are already included in the ordered vertex list. Such a process repeats until all the edges are interrogated (L13, Fig. 7), and the ordered vertex list now becomes $\{a, b, e, f, g, h, i, j, k, l, d, a\}$. As the first and the last vertex is the same, so the last one is removed (L26, Fig. 7), and the final control vertices of the B-Spline curve are $\{a, b, e, f, g, h, i, j, k, l, d\}$, as shown in Fig. 6 (d). Fig. 6 (e) shows the fitted B-Spline curves whose control points are generated from such ordered vertex list, and some concave shaped pores derived from the merged control polygons are clearly seen.

The pore interstices shown in Fig. 6 (e) are also very small. A naïve approach to increase pore interstices is to apply the formulae of Eq.(4) and (5). For concave polygons however, such scaling operation is problematic and this can be clearly seen in Fig. 8 (a), where the scaled point set inside a concave polygon is no longer a subset of the original point set. Fig. 8 (c) shows a problematic case resulted from using the above naïve approach. As highlighted in the circle bounded region, the pores are no longer separated with small interstices, instead overlapped regions appear.

There are several approaches to solving this problem, for instance using the straight skeleton [45] or medial axis transform [46] algorithms. The straight skeleton is created by continuous shrinking of all edges of a polygon at a constant speed, and the skeleton is the set of traces of the moving vertices. The medial axis of a polygon is similar to the straight skeleton but the traces may include parabolic curves. Instead of scaling all the vertices using the polygon centroid as the scaling center, the discussed problem can be resolved by moving the polygon vertices along the straight skeleton or the medial axis. However, both algorithms are relatively complex and the problem can be tackled with an alternative approach. 
To accomplish this, we propose a relatively simpler and more straightforward method as compared with the straight skeleton or medial axis transform algorithms. The idea is very simple: for vertices which are not shared by adjacent Voronoi polygons, for instance $\{a, e, f, g, h, i, k, l\}$ in Fig. 6 (c), the scaled/shrunk vertices are calculated from Eq.(4) and (5). As shown in Fig. 8 (b), the shrunk vertices are now $\left\{a_{1}, e_{1}, f_{1}, g_{1}, h_{1}, i_{1}, k_{1}, l_{1}\right\}$. For vertices which are shared by two Voronoi polygons, for instance $\{b, d, j\}$ in Fig. 6 (c), the vertices are first separately scaled with respect to each child polygon's centroid using Eq.(4) and (5), and the scaled vertices are now $\left\{b_{1}, d_{1}, j_{1}\right\}$ and $\left\{b_{2}, d_{2}, j_{2}\right\}$ in Fig. 8 (b). The coordinates of these scaled vertices $\left\{b_{1}, b_{2}\right\},\left\{d_{1}, d_{2}\right\},\left\{j_{1}, j_{2}\right\}$ are then averaged and output as the new coordinates of the shrunk vertices, i.e. $\left\{b_{12}, d_{12}, j_{12}\right\}$ as shown in Fig. 8 (b). The final vertices of the shrunk polygon are therefore $\left\{a_{1}, b_{12}, e_{1}, f_{1}, g_{1}, h_{1}, i_{1}, j_{12}, k_{1}, l_{1}, d_{12}\right\}$.

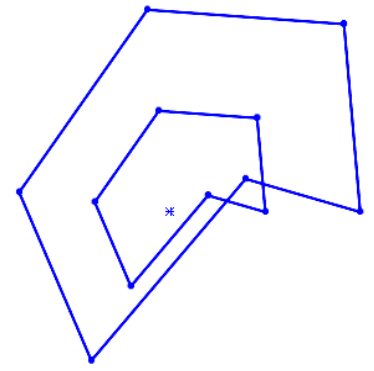

(a)

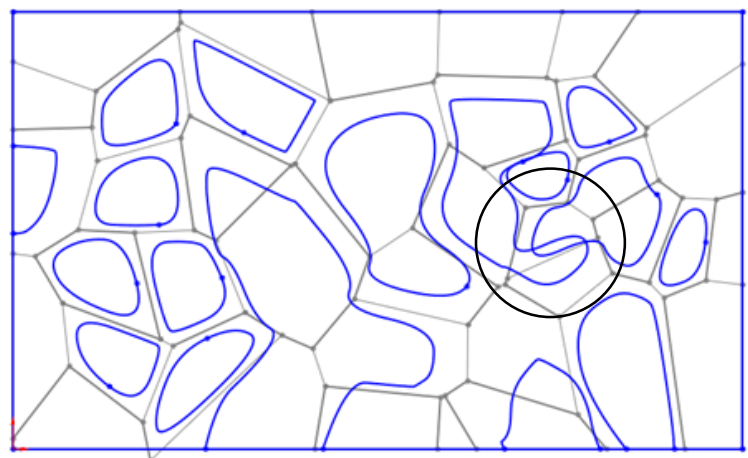

(c)

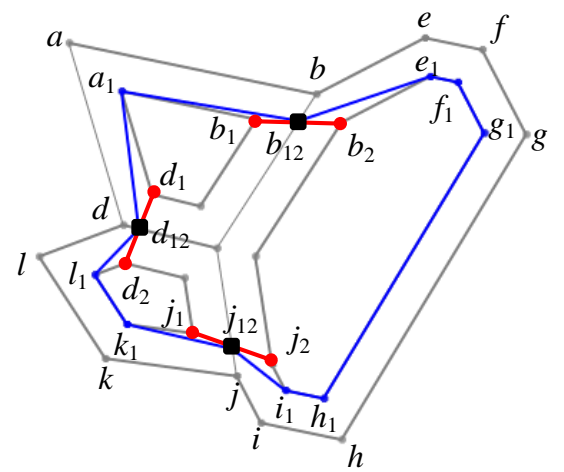

(b)

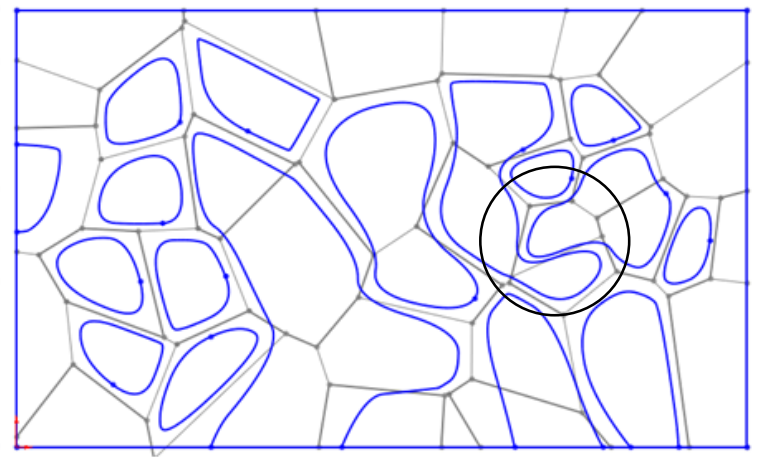

(d)

Fig. 8 Scaling of convex polygons and closed B-Spline fitting. (a) The down-scaled point set is not a subset of the original point set. (b) The proposed polygon shrinking scheme. (c) A problematic case of using naive scaling operation. (d) The desired increment of pore interstices.

Without loss of generality, all the vertices of the shrunk polygon whose vertices are $\left\{v_{0}{ }^{\prime}, v_{1}^{\prime}, \cdots v_{m}^{\prime}\right\}$ can be formulated as:

$$
v_{i}^{\prime}(t)=\sum_{i=0}^{m} w_{i} v_{i}
$$


where $\left\{v_{0}, v_{1}, \cdots v_{m}\right\}$ is the vertices of the original polygon, $w_{i}$ is a weight related to the shrinking or scaling factor $t$. A rigorous proof that the shrunk control polygon will always be contained in the original polygon is straightforward and will not be elaborated in this paper. Fig. 8 (d) demonstrated the result of using the proposed approach to increase the pore interstices, and the effectiveness is clearly shown in contrast with the result shown in Fig. 8 (c).

\subsection{A representation for irregular porous structures}

In Section 3.2 and 3.3, a hybrid Voronoi tessellation and B-Spline based representation is proposed to model irregular-shaped pores. Based on this representation, an irregular porous structure can be defined as follows.

Denote $G_{e x t}$ as the external geometry of an object, and a discrete point set $P=\left\{p_{1}, p_{2}, \cdots, p_{n}\right\}, p_{i} \in G_{\text {ext }}$ includes $n$ points which are inside $G_{e x t}$. Let the Voronoi tessellation of $P$ be $v(P)=\left\{V\left(p_{1}\right), V\left(p_{2}\right), \cdots, V\left(p_{n}\right)\right\}$. For each Voronoi polygon $\delta V\left(p_{i}\right)$ represented by a list of vertices $\left\{v_{0}, v_{1}, \cdots v_{m}\right\}$, the shrunk polygon whose vertices are calculated by Eq. (8) is denoted as $\delta V\left(p_{i}, t\right)$, where $0<t \leq 1$ is the shrinking or scaling factor. The closed B-Spline curve fitted from $\delta V\left(p_{i}, t\right)$ can be obtained from Eq. (2) and denoted as $C\left(p_{i}, t\right)$. We define the point set $S_{i}$ bounded by $C\left(p_{i}, t\right)$ as a pore, and the Boolean union of $S_{i}$ is then modeled as the pore space $S$. A porous structure $\Omega$ is then constructed with the Boolean subtraction of $S$ from $G_{e x t}$ :

$$
\begin{gathered}
S=\bigcup_{i} S_{i} \\
\Omega=G_{e x t} \backslash S
\end{gathered}
$$

where the $U$ and $\backslash$ refer to Boolean set union and subtraction operators.

Fig. 9 illustrates an irregular porous object modeled with the proposed representation. The external geometry $G_{\text {ext }}$ of the porous object is a $2 \mathrm{D}$ rectangle with $0.5 \mathrm{~mm}$ in length and $0.3 \mathrm{~mm}$ in width. One hundred points are randomly generated inside $G_{\text {ext }}$, and ten attributes $\lambda=1,2, \cdots 10$ are randomly associated to the one hundred Voronoi cells.

The aggregated polygons are shown in Fig. 9 (a), and all the polygons are then shrunk with a factor of $t=0.75$ to guarantee sufficient pore interstices. Closed B-Spline curves with order $k=5$ are fitted from the shrunk polygons. The shape and distribution of the pore are depicted in Fig. 9 (b). The results of applying a Boolean subtraction of the pores from the external geometry are shown in Fig. 9 (c), rendered in filled color.

Comparing the irregular porous models shown in Fig. 2 (b) and Fig. 9 (d), it can be clearly seen that they are generically similar in both pore shape and distributions. The difference is that the model in Fig. 2 (b) is reconstructed from SEM images [39], whereas the one shown in Fig. 9 (d) is modelled from a constructive approach using CAD tools. 
The benefit of this constructive design is obvious. Such models are constructed based on stochastic geometry and theoretically there are numerous different configurations in terms of the pore shapes and distributions. Among these infinite choices/configurations, although it is possible to manually design one of them using conventional CAD modelling approaches, however the workload required is hardly comparable with the proposed approach. The manual approach is not only labour-intensive, but also tedious and unwieldy; and moreover the versatility and quality of the design are also closely constrained by designer's creativity and experience.

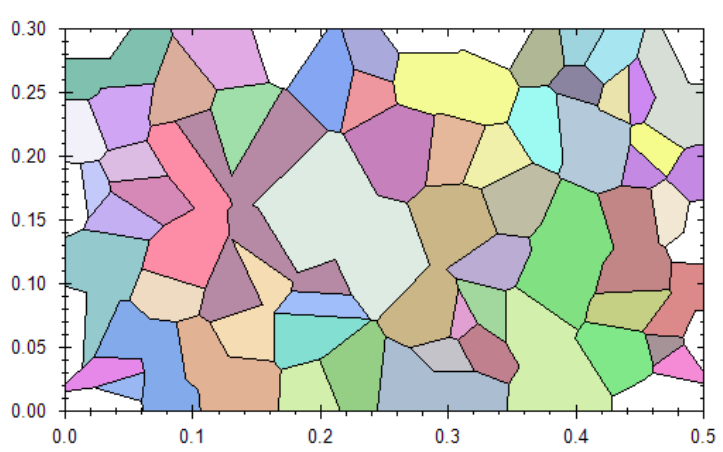

(a)

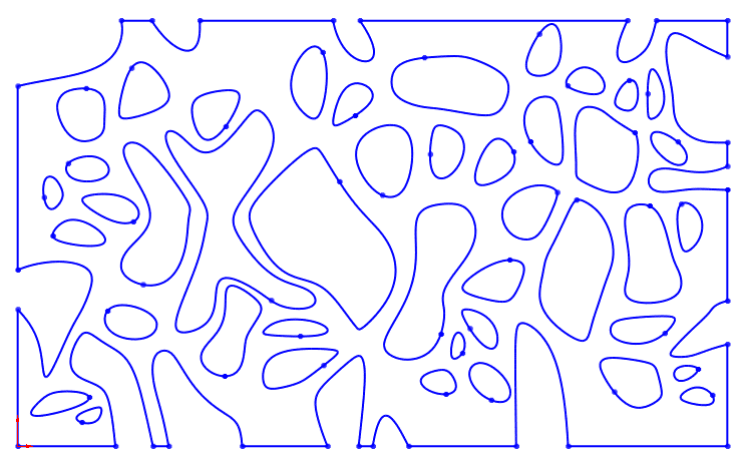

(b)

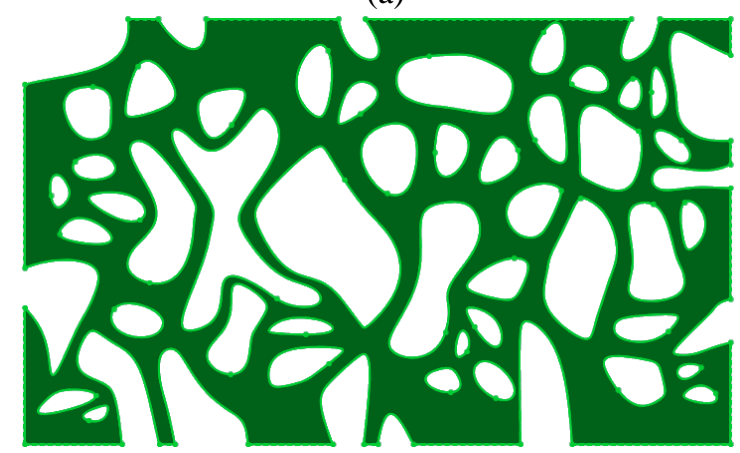

(c)

Fig. 9 An irregular porous structure model. (a) The control polygons used for B-Spline curve fitting. (b) The pore geometries represented by B-Spline curves. (c) Boolean subtraction of the pores from the external geometry and the resultant $2 \mathrm{D}$ representation of the irregular porous structure.

The modelling efficiency of the proposed approach is also an important advantage. If the irregular porous structures are to be designed via optimization methods, for instance, the sensitivity based iterative approaches or population based approaches (e.g. Genetic algorithm), variant geometric models must be efficiently constructed in accordance with parameter updates, otherwise the overall throughputs and productivity of the optimization will be severely undermined.

\section{Implementations and case studies}

Based on the proposed geometric representation, a prototype CAD module is implemented in SolidWorks CAD modeller on Windows 7 platform and Fig. 10 shows a snapshot of this module. In our preliminary work a number of irregular porous object models are constructed and some case studies are offered below to show the application 
and efficacy of the proposed approach.

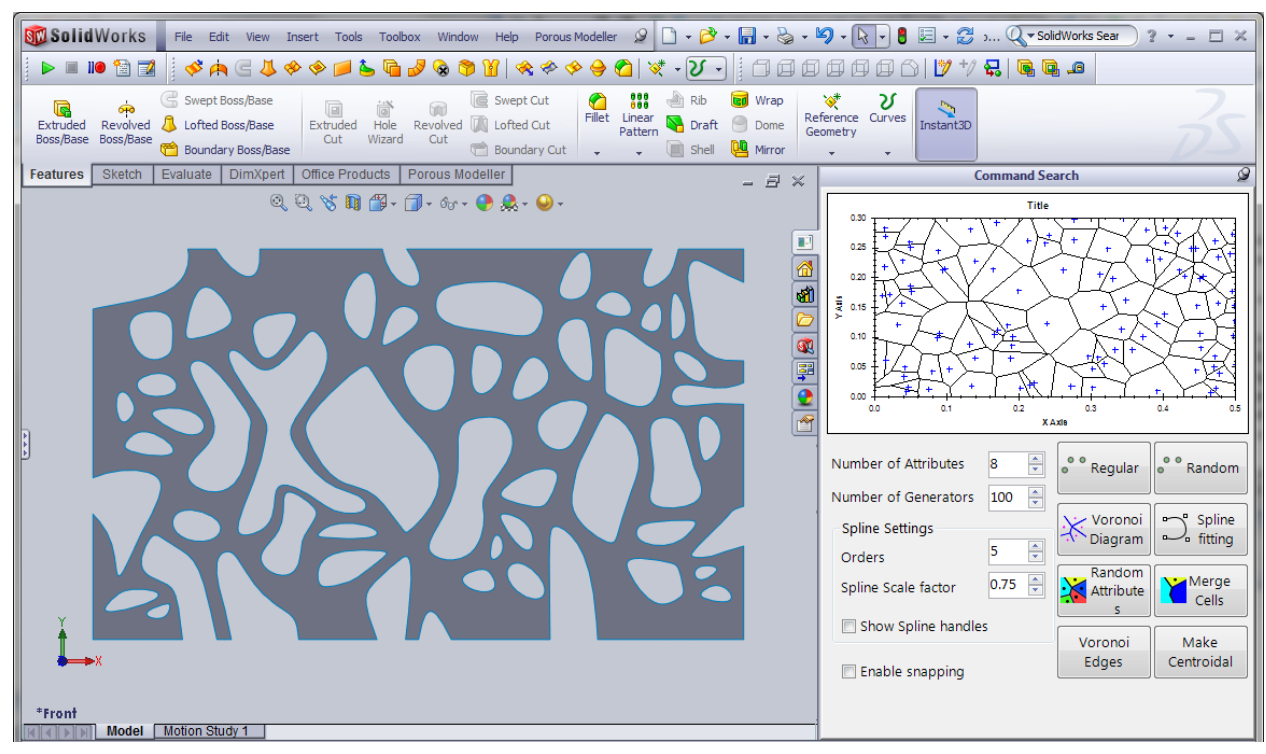

Fig. 10 A snapshot of the prototype porous object modeller.

\subsection{Case study I: Fine tuning pore shapes, topologies and porosities}

As demonstrated in previous sections, the proposed geometric representation has a flexible degree of control on the pore shapes and distributions. Among others, users can accomplish this by (1) Adjusting the count and spatial distribution of generator points; (2) Adjusting the count and value distribution of random attributes associated with Voronoi cells; (3) Tailoring the orders of the B-Spline curve and (4) Tailoring the scaling factors.

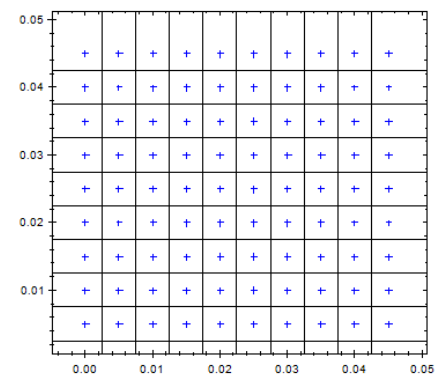

(a)

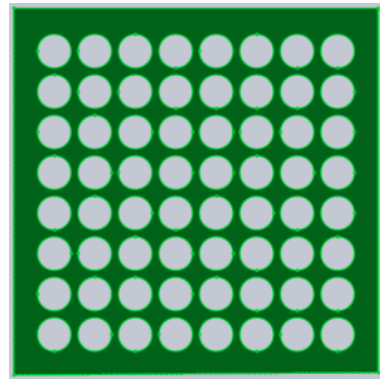

(b)

Fig. 11 A regular distributed generator points and regular shaped pore structure. (a) Generator points on a regular grid. (b) The modelled regular porous objects.

Fig. 11 shows a list of generator points on a regular grid and the resultant porous structure is actually a regular porous structure similar to the one shown in Fig. 1 (a).

Fig. 12 shows two porous structures with different topologies which are generated with the same set of generator points, but with different attributes associated. In Fig. 12 (a) eight attributes are associated with one hundred Voronoi cells, and the probability that adjacent cells have the same attribute value will be much higher than the case where forty attributes are used, as shown in Fig. 12 (b). Therefore after polygon merging, many 
concave-shaped pores appeared in the porous structure shown in Fig. 12 (a), while most of the pores in Fig. 12 (b) are convex shaped.

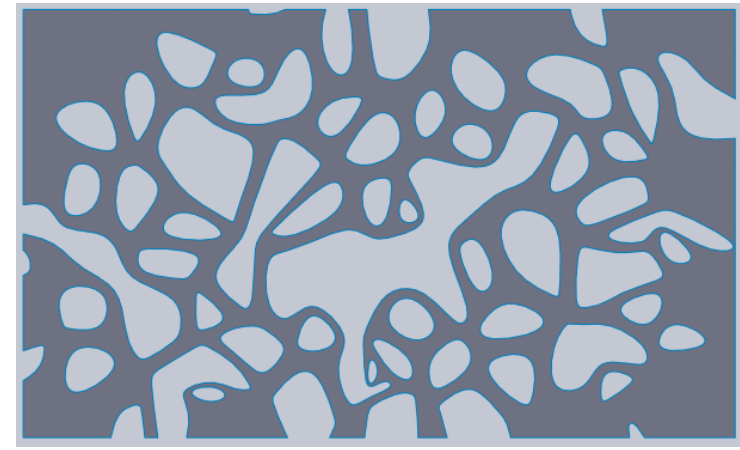

(a)

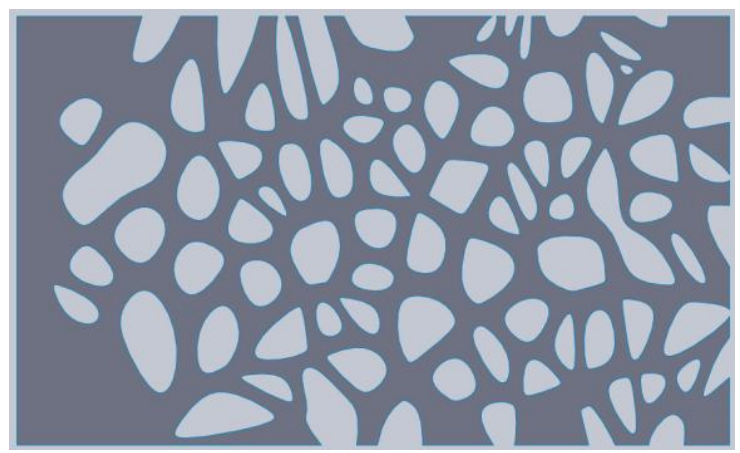

(b)

Fig. 12 Porous structures with different topologies. (a) An irregular porous structure with many concaveshaped pores, the model is constructed with 100 generator points and 8 integer attributes are randomly associated with the Voronoi cells. (b) An irregular porous structure with many convex-shaped pores, the model is constructed with same 100 generator points and 40 integer attributes are randomly associated with the Voronoi cells.

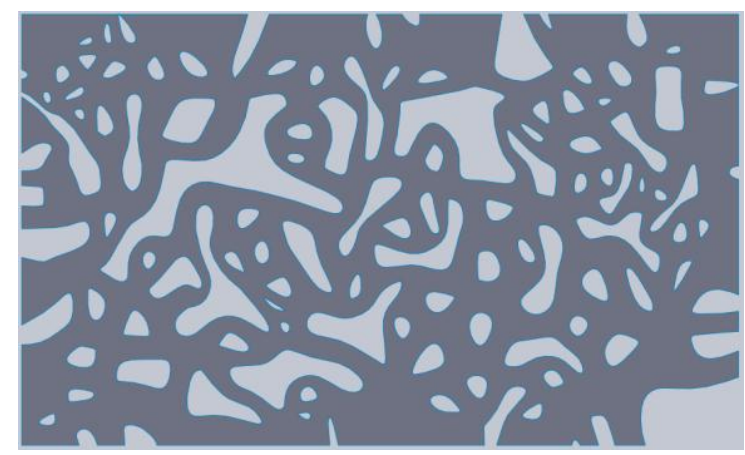

(a)

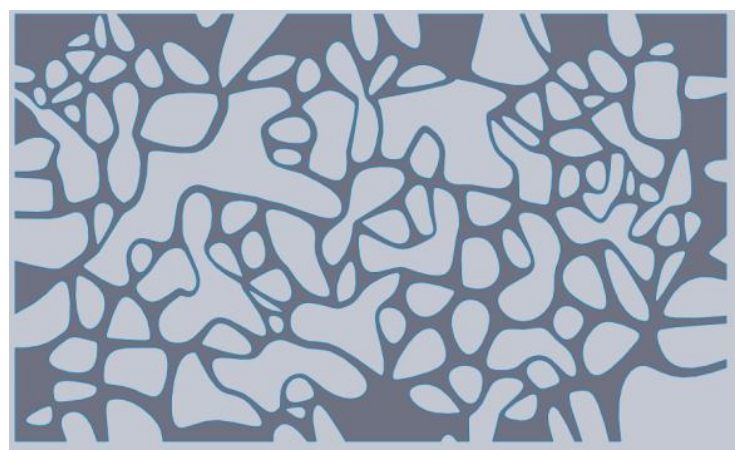

(b)

Fig. 13 Porous structures with different porosities. (a) A porous structure modelled with the scaling factor $t=0.5$. (b) A porous structure modelled with the scaling factor $t=0.85$.

Fig. 13 illustrates two examples with similar shapes but different porosities. Both porous structures are generated using the same set of generator points and attributes, but different scaling factors are used. The porosities of the structures shown in (a) and (b) are $37.5 \%$ and $70.1 \%$ respectively.

\subsection{Case study II: Finite element analysis of a porous structure}

This example examines porous media flow in the interstices of a porous media and a Navier-Stokes analysis for the fluid velocity field is conducted. The original finite element analysis was first conducted by Sirivithayapakorn and Keller [39] at the University of California, Santa Barbara, and they used the structure shown in Fig. 2 (b) as the geometric model. To produce the model geometry they scanned electron microscope images and transferred these images to DXF files and finally the CAD models are analyzed with FEA software. Different from their geometric model, an irregular porous structure is designed using the proposed constructive method, as shown in Fig. 14 (a). 
To compare the results of our model with Sirivithayapakorn and Keller's, all the boundary conditions and physical settings in these two analyses are intentionally kept identical: water moves from right (inlet, with a pressure $p_{0}=0.715 \mathrm{~Pa}$ ) to left (outlet, $p_{1}=0$ $\mathrm{Pa}$ ) across the porous structure and the flow is assumed laminar in the pores and does not enter the grains. Symmetric conditions are imposed on the top and bottom boundaries, and all the remainder pore boundaries are assumed as walls where water does not penetrate.

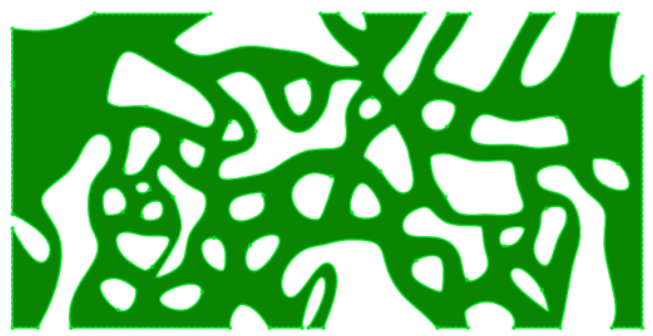

(a)

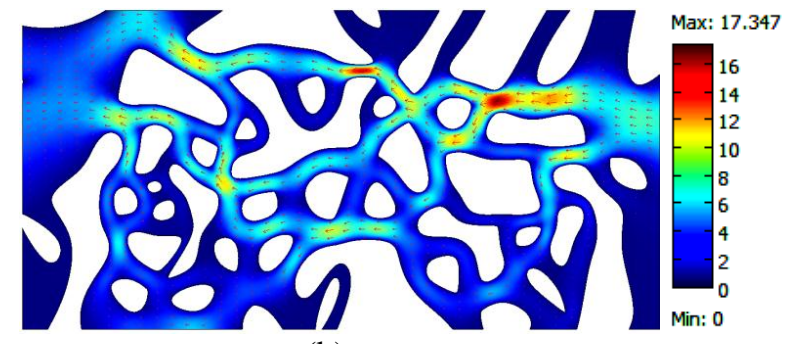

(b)

Fig. 14. A finite element analysis case study. (a) The geometry of a designed irregular porous structures. (b) The flow velocity fields $(\mu \mathrm{m} / \mathrm{s})$ obtained from finite element analysis.

Fig. 14 (b) showed the FEA result of the flow velocity field. The results obtained in our study are consistent with Sirivithayapakorn and Keller's work [39], where water moves faster around the narrowest matrices than other places elsewhere. This study and the obtained results validated the correctness and practical usefulness of the proposed representation. With the proposed design method, such irregular porous structures can be easily designed at interactive time. Moreover, almost infinite geometries and topologies can be modelled, yet no existing porous objects are required to serve as the "templates" to reconstruct from.

\subsection{Case study III: Modeling simple 3D porous solids}

Using the proposed representation and design method, we further modelled two simple 3D porous structures as shown in Fig. 15. The one shown in Fig. 15 (a) is a 3D feature extruded from a 2D porous structure, and the detailed modelling scheme can be found in previous sections. Fig. 15 (b) shows an interesting hollowed cylinder. To construct such a model, a 2D porous surface which is similarly constructed as the one shown in Fig. 14 (a) is first generated; the surface is then wrapped onto the cylindrical surface of the cylinder. This process is similar to the texture mapping widely used in computer graphics, the sole difference is that it is not the material texture but the pore geometry is wrapped/scribed. An extruded cut is then applied on the scribed sketch to realized the deboss effect.

Fig. 16 (a) shows a simple 3D porous structure modelled using the proposed approach. In this example, 50 random generating points are defined in 3D space, and all the control polyhedra are then shrunk with a scaling factor $t=0.5$. As no attribute is used and no cell merging is applied, therefore all the 3D Voronoi cells are convex. Given such a 3D Voronoi tessellation, subdivision surfaces [47] can be derived using these polyhedra as the control polytopes. Note that Catmull and Clark [48] have showed that such tensor product B-spline surfaces do exist for general polyhedra with arbitrary topological 
meshes. Fig. 16 (b) shows part of the internal geometry of Fig. 16 (a), rendered in sectioned wireframe and shaded view.

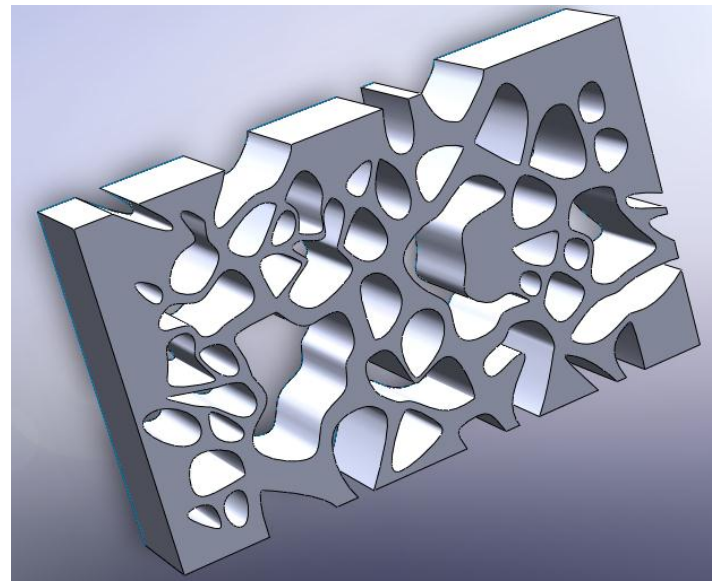

(a)

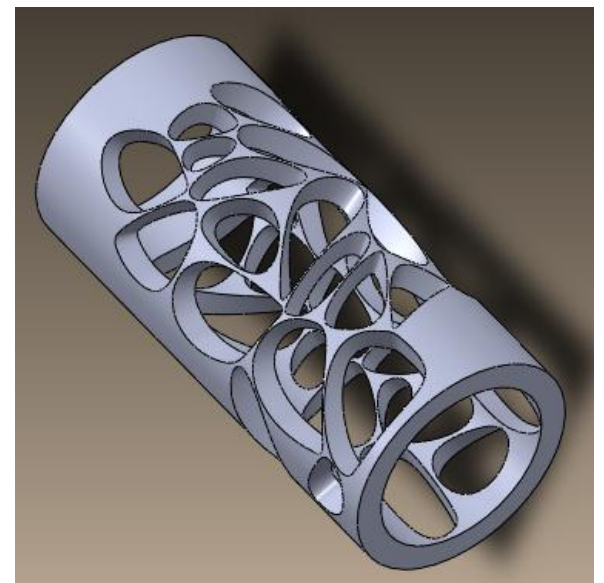

(b)

Fig. 15. Simple 3D porous structures.

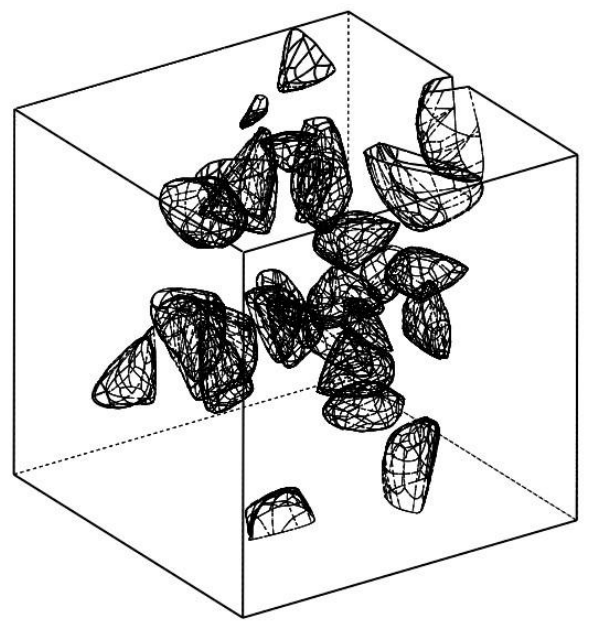

(a)
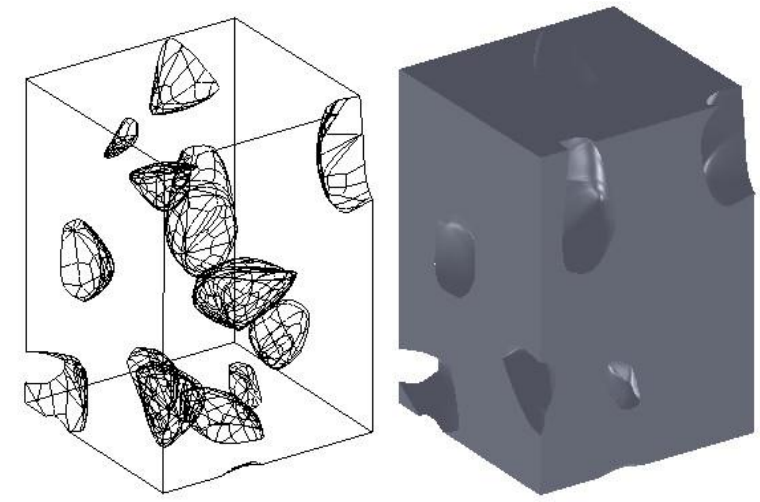

(b)

Fig. 16. A simple 3D porous structure. No attribute is used and no cell merging is applied. (a) Wireframe view of a porous structure generated using 50 random generating points and a scale factor $\mathrm{s}=0.5$; (b) Part of the internal geometry of (a) rendered in sectioned wireframe and shaded view.

Fig. 17 shows 3D aggregated pores with concave shapes. The wireframe view of control polyhedra and the subdivision surfaces are shown in Fig. 17 (a) and (b), and the shaded pore shapes are rendered in Fig. 17 (c). 


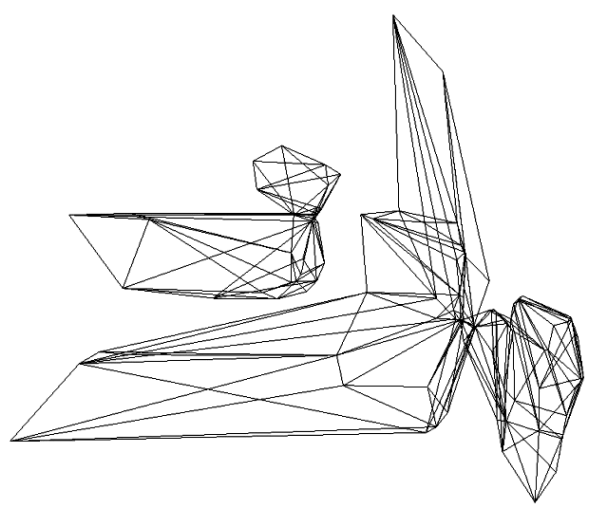

(a)

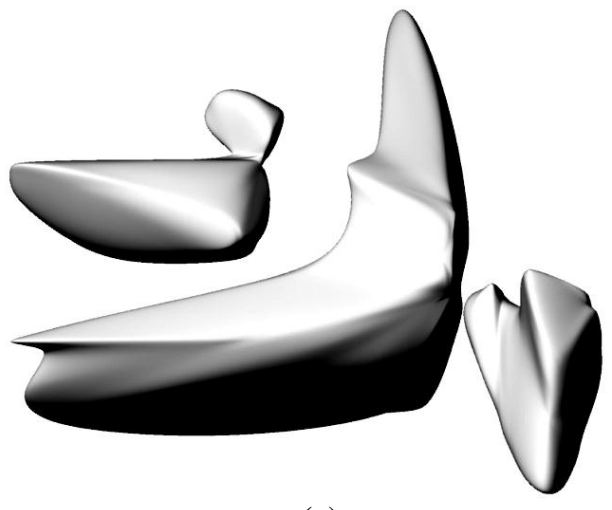

(c)

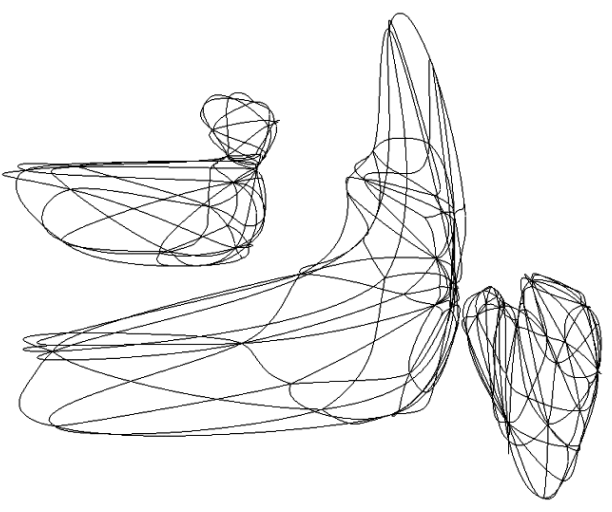

(b)

Fig. 17. 3D geometry of pores with concave shapes. Attributes are used and cell merging is applied. (a) Wireframe view of the merged polygons. (b) Subdivision surfaces of 3D pore geometries (c) Shaded view of the $3 \mathrm{D}$ pore geometries.

\section{Conclusions and Discussions}

A simple and effective geometric representation for irregular porous structure modeling is proposed in this paper. Inspired by the random colloid-aggregation model which explains the formation mechanism of random porous media, Voronoi tessellation is first generated to partition the space into a collection of compartments, selective compartments are then merged together to imitate the random colloid-aggregations. Through this Voronoi cell merging, irregular convex and concave polygons are obtained and the vertices of which are modeled as control points of closed BSpline curves. The fitted B-Spline curves are then employed to represent the boundaries of the irregularshaped pores.

In the past, such irregular porous object models are mostly reconstructed from SEM, $\mathrm{X}$-ray or other microphotographs. Different from such approaches which design irregular porous artifact via exact copy of existing geometries, the proposed representation and modeling method try to mimic the porous structure formation process instead, and the designed structures are therefore independent on any pre-existing objects. This drastically improved the ease of irregular porous structure modeling while at the same time properly maintained the irregularity that are widely found in natural objects. Compared with other 
existing CAD modeling approaches, the proposed approach can easily construct porous structures which look natural and realistic. To our knowledge there seems to be no similar methods that can achieve comparable results using such simple approaches.

We also demonstrated that combining the proposed representation with stochastic geometry, unlimited generically similar, yet geometrically and topologically different porous structures can be designed with little effort. This non-uniqueness and versatility are advantageous instead of limitations since in the real world there are no exactly the same porous structures (e.g. rocks, sandstones and bones etc.) as well.

Given the numerous irregular porous structures of the same gene, however, a legitimate question would be: "Under specific external stimuli and constraints stipulated, how to find the one with the optimal responses in terms of mechanical, thermal, acoustic or other properties"? Theoretically, any population based meta-heuristic optimization schemes [49] such as Genetic Algorithm (GA), Simulated Annealing (SA), Scatter Search (SS), Particle Swarm Optimization (PSO) or Bee Colony (BC) etc. can all be used in conjunction with the proposed representation to optimize the responses or behaviors of the objects. While the optimization problem is interesting in its own right, a thorough investigation is beyond the scope of this paper. An ongoing study on using finite element analysis and particle swarm optimization to optimize an irregular porous structure is under development.

In our future work, we are going to extend the presented representation and use general non-uniform knot vectors to replace the current uniform ones so that pore shapes with sharp features can be properly modeled.

\section{Acknowledgement}

The authors would like to thank the Department of Mechanical Engineering, the University of Hong Kong and the Research Grants Council of Hong Kong SAR Government for supporting this project (HKU7170/06E).

\section{References}

1. Scheffler, M. and P. Colombo, Cellular ceramics: structure, manufacturing, properties and applications. 2005: Vch Verlagsgesellschaft Mbh.

2. Lorna J. Gibson and M.F. Ashby, Cellular solids : structure and properties. 2nd ed. 1997, Cambridge, U.K. ; New York, N.Y. :: Cambridge University Press. xviii, 510 p. :.

3. Degischer, H. and B. Kriszt, Handbook of cellular metals: production, processing, applications. 2002: Vch Verlagsgesellschaft Mbh.

4. $\quad$ Schuth, F., K. Sing, and J. Weitkamp, Handbook of porous solids. 2002: Wiley-Vch.

5. Chua, C.K., et al., Development of a tissue engineering scaffold structure library for rapid prototyping. Part 1: Investigation and classification. International Journal of Advanced Manufacturing Technology, 2003. 21(4): p. 291-301. 
6. Chua, C.K., et al., Development of a tissue engineering scaffold structure library for rapid prototyping. Part 2: Parametric library and assembly program. International Journal of Advanced Manufacturing Technology, 2003. 21(4): p. 302-312.

7. Sun, W., et al., Bio-CAD modeling and its applications in computer-aided tissue engineering. Computer-Aided Design, 2005. 37(11): p. 1097-1114.

8. Vergés, E., et al., 3D reconstruction and quantification of porous structures. Computers and Graphics (Pergamon), 2008. 32(4): p. 438-444.

9. Odgaard, A., Three-dimensional methods for quantification of cancellous bone architecture. Bone, 1997. 20(4): p. 315-328.

10. Hao, L., et al. Modeling bio-scaffolds: Structural and fluid transport characterization based on 3D imaging data. 2009.

11. Hilfer, R. and C. Manwart, Permeability and conductivity for reconstruction models of porous media. Physical Review E - Statistical, Nonlinear, and Soft Matter Physics, 2001. 64(2 I): p. 213041-213044.

12. Manwart, C. and R. Hilfer, Reconstruction of random media using Monte Carlo methods. Physical Review E - Statistical Physics, Plasmas, Fluids, and Related Interdisciplinary Topics, 1999. 59(5 B): p. 5596-5599.

13. Manwart, C., S. Torquato, and R. Hilfer, Stochastic reconstruction of sandstones. Physical Review E - Statistical Physics, Plasmas, Fluids, and Related Interdisciplinary Topics, 2000. 62(1 B): p. 893-899.

14. Guest, J.K. and J.H. Prévost, Optimizing multifunctional materials: Design of microstructures for maximized stiffness and fluid permeability. International Journal of Solids and Structures, 2006. 43(22-23): p. 7028-7047.

15. Hollister, S.J. and N. Kikuchi, A comparison of homogenization and standard mechanics analyses for periodic porous composites. Computational Mechanics, 1992. 10(2): p. 73-95.

16. http://archive.nlm.nih.gov/proj/ttp/flash/hooke/hooke.html.

17. Hollister, S.J., R.D. Maddox, and J.M. Taboas, Optimal design and fabrication of scaffolds to mimic tissue properties and satisfy biological constraints. Biomaterials, 2002. 23(20): p. 40954103.

18. Hutmacher, D.W., Scaffolds in tissue engineering bone and cartilage. Biomaterials, 2000. 21(24): p. 2529-2543.

19. Lal, P. and W. Sun, Computer modeling approach for microsphere-packed bone scaffold. CAD Computer Aided Design, 2004. 36(5): p. 487-497.

20. McIntosh, L., J.M. Cordell, and A.J. Wagoner Johnson, Impact of bone geometry on effective properties of bone scaffolds. Acta Biomaterialia, 2009. 5(2): p. 680-692.

21. Mullen, L., et al., Selective laser melting: A regular unit cell approach for the manufacture of porous, titanium, bone in-growth constructs, suitable for orthopedic applications. Journal of Biomedical Materials Research - Part B Applied Biomaterials, 2009. 89(2): p. 325-334.

22. Fang, Z., B. Starly, and W. Sun, Computer-aided characterization for effective mechanical properties of porous tissue scaffolds. CAD Computer Aided Design, 2005. 37(1): p. 65-72.

23. Hollister, S.J., Porous scaffold design for tissue engineering. Nature Materials, 2005. 4(7): p. 518524.

24. Hollister, S.J., et al., Application of homogenization theory to the study of trabecular bone mechanics. Journal of Biomechanics, 1991. 24(9): p. 825-839.

25. Ko, C.-C., D.H. Kohn, and S.J. Hollister. Characterizing elastic properties of bimaterial interphase composites: comparison of experimental and analytical results. 1994. Chicago, IL, USA: ASME.

26. Borodkin, J.L. and S.J. Hollister. Homogenization and direct estimates of trabecular tissue strains. 1995. Beever Creek, CO, USA: ASME.

27. Hollister, S.J., A homogenization sampling procedure for calculating trabecular bone effective stiffness and tissue level stress. Journal of Biomechanics, 1994. 27(4): p. 433-444.

28. Hollister, S.J. and N. Kikuchi, Homogenization theory and digital imaging: A basis for studying the mechanics and design principles of bone tissue. Biotechnology and Bioengineering, 1994. 43(7): p. 586-596.

29. Fyhrie, D.P., et al., Predicting trabecular bone strength and micro-strain using homogenization theory. Journal of Biomechanics, 1989. 22(10): p. 1014. 
30. Khatam, H. and M.-J. Pindera, Parametric finite-volume micromechanics of periodic materials with elastoplastic phases. International Journal of Plasticity, 2009. 25(7): p. 1386-1411.

31. Porfiri, M., N. Nguyen, and N. Gupta, Thermal conductivity of multiphase particulate composite materials. Journal of Materials Science, 2009. 44(6): p. 1540-1550.

32. Lin, C.Y., N. Kikuchi, and S.J. Hollister, A novel method for biomaterial scaffold internal architecture design to match bone elastic properties with desired porosity. Journal of Biomechanics, 2004. 37(5): p. 623-636.

33. Schroeder, C., et al., Computer-aided design of porous artifacts. CAD Computer Aided Design, 2005. 37(3): p. 339-353.

34. Luchnikov, V.A., et al., Voronoi-Delaunay analysis of voids in systems of nonspherical particles. Physical Review E, 1999. 59(Copyright (C) 2009 The American Physical Society): p. 7205.

35. Rigby, S.P. and L.F. Gladden, NMR and fractal modelling studies of transport in porous media. Chemical Engineering Science, 1996. 51(10): p. 2263-2272.

36. Mohebi, A., P. Fieguth, and M.A. Ioannidis, Statistical fusion of two-scale images of porous media. Advances in Water Resources, 2009. 32(11): p. 1567-1579.

37. Tsakiroglou, C.D., et al., A new approach for the characterization of the pore structure of dual porosity rocks. Chemical Engineering Science, 2009. 64(5): p. 847-859.

38. Wong, K.H., et al., Honeycomb structured porous films from amphiphilic block copolymers prepared via RAFT polymerization. Polymer, 2007. 48(17): p. 4950-4965.

39. Sirivithayapakorn, S. and A. Keller, Transport of colloids in saturated porous media: A pore-scale observation of the size exclusion effect and colloid acceleration. Water Resources Research, 2003. 39(4).

40. Virnovsky, G.A., A. Lohne, and O.I. Frette, Modeling capillary pressure using capillary bundles with arbitrary cross-sections obtained from photomicrographs. Journal of Petroleum Science and Engineering, 2009. 69(1-2): p. 117-128.

41. Levy, R.A., et al., CT-generated porous hydroxyapatite orbital floor prosthesis as a prototype bioimplant. American Journal of Neuroradiology, 1997. 18(8): p. 1522-1525.

42. Schaefer, D.W. and K.D. Keefer, Structure of random porous materials: Silica aerogel. Physical Review Letters, 1986. 56(20): p. 2199-2202.

43. $\quad$ Okabe, A., et al., Spatial Tessellations: Concepts and Applications of Voronoi Diagrams. 2000: Wiley.

44. Fortune, S., A sweepline algorithm for Voronoi diagrams, in Proceedings of the second annual symposium on Computational geometry. 1986, ACM: Yorktown Heights, New York, United States. p. 313-322.

45. Aichholzer, O., et al., A novel type of skeleton for polygons. Journal of Universal Computer Science, 1995. 1(12): p. 752-761.

46. Blum, H., A transformation for extracting new descriptors of shape. Models for the perception of speech and visual form, 1967. 19(5): p. 362-380.

47. Ma, W., Subdivision surfaces for CAD--an overview. Computer-Aided Design, 2005. 37(7): p. 693-709.

48. Catmull, E. and J. Clark, Recursively generated B-spline surfaces on arbitrary topological meshes. Computer-Aided Design, 1978. 10(6): p. 350-355.

49. Talbi, E.-G., -, Metaheuristics : from design to implementation. 2009, Hoboken, N.J. :: John Wiley \& Sons. xxix, 593 p. :. 